\title{
Changing Trends of Adult Lymphoma in the Kingdom of Saudi Arabia - Comparison of Data Sources
}

\author{
Muhammad Shahzad Rauf*, Saad Akhtar, Irfan Maghfoor
}

\begin{abstract}
Background: Lymphoma is one of the most common malignancies affecting the young Saudi population. This disease has diversified pathologies and clinical stages that necessitate well optimized clinical management. Regular updates of epidemiological behavior of lymphoma from various parts of the world are available but studies from the Kingdom of Saudi Arabia (KSA) in this field are not consistent. Objectives: The aim of this study was to investigate the current trends in presentation and distribution of lymphoma with special reference to incidence and mortality, gender, age, histopathological subtypes, and clinical stages at King Faisal Specialist Hospital and Research Centre (KFSH\&RC). Materials and Methods: Our study included lymphoma data from Saudi Cancer Registry, and relative comparison against KFSH\&RC tumor registry data, Gulf country data and International Agency for Research on Cancer data. Results: Common tumors in the West (lung, colon, and prostate) were found to be much less frequent in KSA while leukemia, lymphoma and thyroid cancers were more common. Non-Hodgkin Lymphoma (NHL) ranked 3rd most common cancer with age-adjusted incidence of 6/100,000. Estimated age adjusted mortality was 4/100,000 in KSA. There was a peak rise in incidence of lymphoma in 1997-2007. Most common NHL was diffuse large B cell lymphoma at KFSH\&RC. A total of 434 cases were diagnosed in 5 years with $55 \%$ of them at advanced stage and $35 \%$ demonstrating bulky disease and high risk. KFSH\&RC registered $35 \%$ of Hodgkins and $21 \%$ of total NHL identified in entire Saudi Cancer Registry, 2009. Conclusions: Results of this study are very unique, and reveal diverse trends. The findings provide valuable insights in the understanding of current epidemiological features of lymphoma in this part of the world.
\end{abstract}

Keywords: Lymphoma - Kingdom of Saudi Arabia - epidemiology - Saudi cancer incidence and mortality

Asian Pac J Cancer Prev, 16 (5), 2069-2072

\section{Introduction}

As per International Agency for Research on Cancer (IARC 2012) there were estimated 14.1 million cancer cases around the world in 2012, of these, 7.4 million cases were in men and 6.7 million in women. This number is expected to rise to 24 million cases by 2035 . Non-Hodgkin lymphoma (NHL) ranked 10th most common cancer contributing 386000 new cases in 2012 constituting $2.75 \%$ of all cancer. Hodgkin lymphoma (HL) ranked 25th most common cancer in 2012 constituting $0.5 \%$ of all cancer. Lymphoid malignancies composed of variety of different morphologic and clinical syndromes.

\section{Materials and Methods}

We conducted a retrospective, descriptive, epidemiologic analysis of presentation, distribution incidence and mortality of lymphoma at King Faisal Hospital and Research Centre (KFSH\&RC tumor registry 2012) data established in 1975 . We also performed their relative comparison against, Saudi cancer registry data (SCR 2009; 2010), which is a population-based registry established in 1992, gulf countries data and IARC data. We also did brief retrospective analysis outcome of NHL presented to KFSH\&RC and its comparison against international trials. All patients and / or their guardians provided informed consent for all treatments, procedures as per institutional requirements. The study conformed to the ethical guidelines of the World Medical Association Declaration of Helsinki. Kaplan-Meier method was used to do survival analysis.

\section{Results}

KFSH\&RC is a tertiary care hospital for oncology and the foremost center for cancer therapy in KSA opened in June 1975. Tumor registry databases now include 75,233 malignant cases seen from 1975-2012. For each newly identified lymphoid neoplasm, patient information like, patient demographic data, including age and sex, and information on the tumor histologic type, primary site, 
Muhammad Shahzad Rauf et al

type of treatment and immunophenotype were collected.

Based on trends in crude relative frequency, breast cancer led the list of total cancer cases seen followed by leukemia; NHL is $3^{\text {rd }}$ commonest cancer whereas HL is 8th commonest cancer seen at KFSH\&RC in adult patients.

\section{NHL and HL}

\section{Frequency distribution}

1975-2012: NHL is 3rd commonest cancer since 1975-2012 as per tumor registry data. 5652 cases have been registered at $\mathrm{KFSH} \& \mathrm{RC}$, constituting $7.5 \%$ of all cancers. HL is 8 th commonest cancer with 3020 cases, constituting $4.0 \%$ of all cancers registered. If we compare 2009 and 2010 SCR and KFSH\&RC tumor registry 2009 , KFSH\&RC registered $35 \%$ of Hodgkin's and $21 \%$ NHL identified in entire SCR 2009. Over the last decade there has been rapid rise in lymphoma cases registered at KFSH\&RC but in last five years incidence of lymphoma have been stable (Figure 1).

2008-2012: 1345 cases were diagnosed as Lymphoma, 790 diagnosed to have NHL with male to female ratio 1.3:1. In 2012, NHL account for $5.7 \%$ of all cancer cases. It is the 3rd commonest malignancy in male and 6th commonest in female adult. 555 patients were found to have HL with male to female ratio 1.4 to 1 .Also in $2012 \mathrm{HL}$ is the 11th commonest cancer in male and 7th commonest in female adults. In the U.S.A., it constitutes $0.6 \%$ of all cancers, compared to $3.8 \%$ of all cancers at KFSH\&RC in 2012.

\section{Age distribution}

1975-2012: NHL patients registered, with peak incidence between 40-60 years of age whereas HL with peak incidence between 15-39 years as shown in Table 1.1.

2008-2012: Both HL \& NHL patients registered, with peak incidence between 15-39 years of age, as shown in table 1.2.

\section{Pathological distribution}

From 2008-2012, out of 1345 cases, $41 \%$ (555) of patients were diagnosed to have HL and 59\% (790) found to have NHL. $49 \%$ of all lymphomas are B-cell lymphoma and T-cell lymphomas represents $10 \%$ of all lymphomas. $83 \%$ of all NHL are B cell lymphoma and $17 \%$ of NHL are $\mathrm{T}$ cell lymphoma. $58 \%$ of B cell Lymphomas were diagnosed, as diffuse large B cell lymphoma (DLBL).

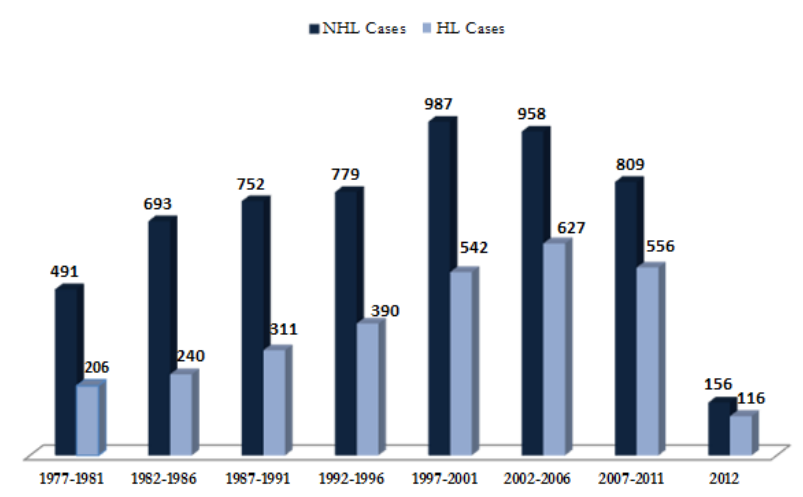

Figure 1. Lymphoma Cases Trend Since 1975-2012. Source: Tumor Registry Data 2012 Published with permission
Table 1.1 Age Distribution 1975-2012

\begin{tabular}{lcclcccc}
\hline Cancer & Age Group & No & $\%$ & Cancer & Age Group & No & $\%$ \\
\hline NHL & $00-14$ & 856 & 15.1 & & $00-14$ & 891 & 29.5 \\
& $15-39$ & 1512 & 26.8 & & $15-39$ & 1595 & 52.8 \\
& $40-59$ & 1816 & 32.1 & HL & $40-59$ & 1209 & 13.2 \\
& $>60$ & 1468 & 26 & & $>60$ & 1348 & 4.5 \\
\hline
\end{tabular}

Table 1.2. Age Distribution 2008-2012

\begin{tabular}{lccccccc}
\hline Cancer & Age Group & No & $\%$ & Cancer & Age Group & No & $\%$ \\
\hline NHL & $0-14$ & 92 & 11.6 & $0-14$ & 133 & 23.9 \\
& $15-39$ & 250 & 31.6 & & $15-39$ & 343 & 61.8 \\
& $40-59$ & 235 & 29.7 & HL & $40-59$ & 59 & 10.6 \\
& $>60$ & 213 & 26.9 & & $>60$ & 20 & 3.6 \\
\hline
\end{tabular}

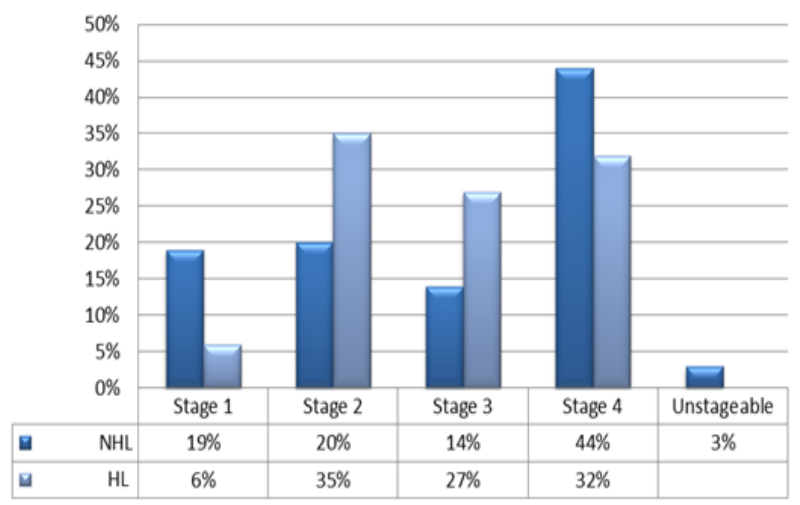

Figure 2. Stage Distribution of Analytic Lymphoma Cases from 2008-2012. Source: Tumor Registry Data 2012 Published with permission

Follicular lymphoma represents $9 \% .41 \%$ of $\mathrm{T}$ cell lymphoma were Mycosis fungoides. $32 \%$ of lymphomas involved extra nodal tissue. $67 \%$ of HL was nodular sclerosis type.

\section{Stage distribution}

From 1975-2012, 50.4\% NHL were advance stage and from 2008-2012, based on analytic cases following are stage distribution with details. We can see $32 \%$ of HL and $44 \%$ of NHL presented as advanced stage as shown (Figure 2).

Diffuse Large B Cell Lymphoma and its Outcome at $K f \operatorname{sh} \& R c$

\section{Incidence/Prevalence}

434 new cases of diffuse large B cell lymphoma (DLBL) were diagnosed from 2003-2007, with male to female ratio $\mathrm{M}$ : F was 1.3:1. Based on staging, number of patients diagnosed with Stage 1; 63(14.51\%), stage 2; 117 (26.95\%), stage 3; 65 (14.97\%) stage $4 ; 173(39.86 \%)$ unknown; $16(3.68 \%) .152(35 \%)$ patients were diagnosed as bulky disease. 299 (69\%) patients had extra nodal involvement. Most common extra nodal involvement site found to be gastric (59 patients) and bone (49 patients). So almost $55 \%$ of patients diagnosed as advanced stage, $35 \%$ had bulky disease. $16 \%$ had high International Prognostic Index (IPI). But if we review (Ziepert et al., 2010) analyses from 1,062 patients treated with R-CHOP in three prospective clinical trials (MInT, 380 patients; 
Table 2. Estimated Incidences and Mortality of NHL in KSA and Relevant Other Countries in 2012

\begin{tabular}{lcc}
\hline Country & ASR per 100,000 & ASM per 100,000 \\
\hline Israel & 16.2 & 4.1 \\
USA & 12.3 & 3.5 \\
Australia & 12.2 & 3 \\
UK & 10.1 & 2.9 \\
Lebanon & 13 & 7.5 \\
KSA & 6.3 & 4.1 \\
Qatar & 6.3 & 4.5 \\
Kuwait & 6 & 2 \\
UAE & 5.8 & 4.3 \\
Bahrain & 4.2 & 0.6 \\
\hline
\end{tabular}

Table 3. Estimated Incidences and Mortality of HL in KSA and Relevant Other Countries in 2012

\begin{tabular}{lcc}
\hline Country & ASR per 100,000 & ASM per 100,000 \\
\hline Israel & 3.7 & 0.3 \\
USA & 2.5 & 0.3 \\
Australia & 2.4 & 0.2 \\
UK & 2.3 & 0.3 \\
Lebanon & 3.7 & 1.6 \\
KSA & 1.6 & 0.8 \\
Qatar & 1.4 & 0.2 \\
Kuwait & 1.5 & 0.1 \\
UAE & 0.9 & 0.3 \\
Bahrain & 1.1 & 0.1 \\
\hline
\end{tabular}

Mega CHOEP, 72 patients; RICOVER-60, 610 patients). 44 percent patients were advanced stage, $10 \%$ were found to be in high risk IPI category. $17 \%$ had more than one extra nodal involvement.

\section{Treatment outcome}

198 patients treated with R-CHOP from 2003-2007 at KFSH\&RC. At a median follow-up for survivors of 68 months (19-102 months), event-free survivals (EFS) and overall (OS) were 55\%, 64\% respectively. EFS and OS for four groups according to IPI were 79\%, 46\%, 47\%, $29 \%$ and $85 \%, 59 \%, 53 \%, 36 \%$ for low, low intermediate, high intermediate and high-risk groups respectively. In Ziepart et al analysis The IPI score was highly significant for the EFS, PFS, and OS end points, 3-year rate estimates for EFS, PFS, and OS: IPI 0 and 1: $81.3 \%, 87.0 \%$, and 91.4\%; IPI 2: $68.5 \%, 74.7 \%$, and $80.9 \%$; IPI $3: 53.2 \%$, $58.6 \%$, and $65.1 \%$; IPI 4 and $5: 49.5 \%, 55.8 \%$, and $59.0 \%$ respectively, when all 1,062 patients were analyzed as a single data. But 0nly 10 percent were found to be in high IPI category. 17\% had more than one extra nodal involvement. Definition of bulky disease is variable so we could not do analyses.

\section{Discussion}

IARC estimated in 2012 that the worldwide agestandardized incidence rate (ASR) per 100,000 Population of NHL was 5.1, higher in men (ASR 6.0/100000) then women (ASR 4.1/100000). Israel with ASR of 16.2 found to have highest incidence of NHL. ASR in USA was 12.3. Incidence increased in many high-income countries between 1950s and 1990s, but no further increase has been observed during the past decade. Worldwide AgeStandardized mortality (ASM) was 2.5 per 100,000 populations. In the United States, ASM of NHL was found to be 3.5. World ASR of HL was 0.9. Again highest incidence found to be in Israel that is $3.7 / 100000$. World ASM was 0.3/100000. If we review Saudi cancer registry, from January 012009 till December 31 2010, total numbers of cancer cases reported to the SCR were 26960. NHL was 3rd commonest cancer constituting 7.2\% of all cancer. HL was 8 th commonest cancer constituting $3.5 \%$ of all cancers. NHL was one of the most prevalent types of cancer in KSA. In year 2009 and 2010, there were 1412 cases of adult NHL analyzed by SCR accounting for $7.2 \%$ of all newly diagnosed cancers. This cancer ranked second among male population and fourth among female population. It affected $829(59 \%)$ males and 583 (41\%) females. Overall ASR was 6/100,000 in 2009 and $5.7 / 100,000$ in 2010. ASR for males was 6.8 in 2009 and 6.2 in 2010 and 5.1 for females in 2009 and 2010. The median age at diagnosis was 53 years. $16.5 \%$ were diagnosed to have localized NHL whereas $17.6 \%$ regional and $41.9 \%$ as distant disease and $24 \%$ were unknown stage. Based on SEER data (SEER 2014) from 2004-2010, $28 \%$ of patients were diagnosed as localized and $49 \%$ diagnosed as distant disease.

Estimated ASR as per IARC 2012 of NHL in KSA is relatively higher than in some Gulf countries like United Arab Emirates, Bahrain and Kuwait. Despite the low ASR of NHL cancer in Saudi Arabia compared to western countries, the ASM of NHL remained slightly high. For example, the ASM of NHL in Australia, UK and USA were lower than KSA as shown in Table 2

In year 2009 and 2010, there were 680 cases of adult HL analyzed by SCR accounting for $3.5 \%$ of all newly diagnosed cases. It affected 388 (56\%) males and 292 (44\%) females. The overall ASR was $2 / 100,000$ in 2009 and 1.9 in 2010. ASR was 2.3 in 2009 but was 2.1 in 2010 for males and 1.7 for females in both years. The median age at diagnosis was 25 years. $14 \%$ were diagnosed to have localized HL whereas $25 \%$ regional and $40 \%$ as distant disease. $21 \%$ were unknown stage. Based on SEER data from 2004-2010, 38\% of patients diagnosed as distant disease.

Estimated ASR as per IARC 2012 of HL in KSA is relatively higher than in some Gulf countries like United Arab Emirates, Bahrain and Kuwait. Despite the low ASR of HL cancer in Saudi Arabia compared to western countries, the ASM of NHL remained slightly high. For example, the ASM of NHL in Australia, UK, USA and other Gulf countries were lower than KSA as shown in Table 3 .

In our study HL represents $41 \%$ of all lymphomas at KFSHRC which is lot higher than western countries which ranges between $5-15 \%$ whereas $\mathrm{T}$ cell lymphoma incidence is also relatively higher than western countries which ranges from $5-10 \%$ in western countries

In conclusion, our data clearly shows lymphoma remains one of the leading cancers in KSA and forms a significant proportion of new cancer patients referred to KFSH\&RC. Crude relative frequencies of primary cancers seen at KFSH\&RC are very different from the 
Western world. Common tumors of the West (lung, colon, and prostate) are much less frequent here at KSA while leukemia; lymphoma and thyroid cancers are more common. There has been peak rise in incidence of lymphoma in 1997-2007, but incidences have been stable in last 5 years. This rise has been noted worldwide, in both genders, and increase in high-grade NHL is predominant. This could be due to reasons proven already by epidemiological studies confirming that modifiable risk factors, including obesity, lack of physical activity, high consumption of meat, are positively linked with NHL. There are also non-modifiable risk factors that can increase the risk of NHL, which includes age, sex, etc. (Skibola CF 2007). Various studies have been published regarding Asian continent, to explain different subtype distribution among different countries. The association between lymphomas and viral infections has been discussed, especially regarding Epstein Barr Virus infection in Hodgkin lymphoma patients (Fatima et al., 2011). Risk factors have been detected such as a higher consumption of carbohydrate in Oman (Ali et al., 2013) and cigarette smoking, consumption of mutton and consumption of milk in India. (Balasubramaniam et al., 2013). These mentioned risk factors are different in various parts of the KSA and world resulting diverse distribution of lymphoid malignancy. Alarming feature is the ASM of lymphoma in $\mathrm{KSA}$ is higher as compare to few gulf countries.

It is also evident that lymphoma patients presented at KFSH\&RC are more advanced staged involving younger population and relatively have more extra nodal involvement, because of these bad prognostic features survival at KFSH\&RC is lower as compare to survival data reported in various international studies.

Prevention of Lymphoma is vital subject among the $\mathrm{KSA}$ population. In this region, it has many epidemiologic and clinical features that are different from those in the rest of the world. Furthermore, relatively young population being involved with cancer and large discrepancies in the availability of resources at diagnostic and treatment levels. It is extremely important to have regional guidelines on cancer care, for lymphoid malignancies.

\section{Acknowledgements}

We are thankful to Saudi Cancer Registry, KFSH\&RC Tumor Registry for allowing us to use their data. We are also thankful to Asif Mehmood, Tumor Registrar for collecting the data. No financial interest is being involved.

\section{References}

Ali A, Al-Belushi BS, Waly MI, et al (2013). Dietary and lifestyle factors and risk of non-Hodgkin's lymphoma in Oman. Asian Pac J Cancer Prev, 14, 841-8.

Balasubramaniam G, Saoba S, Sarade M, et al (2013). Casecontrol study of risk factors for Non-Hodgkin lymphoma in Mumbai, India. Asian Pac J Cancer Prev, 14, 775-80.

Fatima S, Ahmed R, Ahmed A (2011). Hodgkin lymphoma in Pakistan: an analysis of subtypes and their correlation with Epstein Barr virus. Asian Pac J Cancer Prev, 12, 1385-8

Globocan, The global cancer atlas. Available from: http:// globocan.iarc.fr/ia/World/atlas.html. Accessed in June 2014.
King Faisal Specialist Hospital and Research Centre (2011). Tumor Registry Annual Report. Riyadh: Tumor Registry; Accessed June 10, 2014.

Saudi Cancer Registry (2009). Cancer incidence report in Saudi Arabia. Riyadh: Ministry of Health.

SEER Cancer Statistics Factsheets:National Cancer Institute. Bethesda, MD, http://seer.cancer.gov/statfacts/html. Accessed in June 2014.

Skibola CF (2007). Obesity, diet and risk of non-Hodgkin lymphoma. Cancer Epidemiol Biomarkers Prev, 16, 392-5.

Ziepert M, Hasenclever D, Kuhnt E, et al (2010). Standard International prognostic index remains a valid predictor of outcome for patients with aggressive CD20+ B-cell lymphoma in the rituximab era. J Clinical Oncol, 28, 2373-80. 